\title{
Clinical burden of viral sexually transmitted infections in adolescents and young adults with HIV attending an urban clinic in sub-Saharan Africa
}

\begin{abstract}
Viral sexually transmitted infections (STIs) are highly prevalent among people infected with HIV. Diagnosis of viral STIs in sub-Saharan Africa is still a challenge because of the high viral test costs involved and as a result, no published data exist on the burden of viral STIs among ALHIV and YALHIV in sub-Saharan Africa. This study aimed to provide data on the clinical burden of viral STI consultations among ALHIV and YALHIV in a resource-limited setting.

Overall, 165 HIV positive adolescents and young adults accessed treatment from our transition STI program. Eightyone $(49.1 \%)$ patients were diagnosed with viral STIs. Herpes simplex virus (HSV) infection was the predominant viral STI (70.4\%) followed by Human Papilloma Virus (HPV) infection (Condylomata acuminata) (35.8\%) and Molluscum contagiosum (MCV) (4.9\%). Clinically diagnosed herpes simplex viral infections were the predominant sexually transmitted infection in our adolescent and young adult HIV cohort in a large urban HIV clinic in sub-Saharan Africa. Clinicians should maintain a high index of suspicion for the presence of genital HSV infections among symptomatic HIV positive adolescents and young adults. Opportunities directed at developing single, dual or multiple point-of-care viral STI testing in line with diagnosing asymptomatic viral STIs in this population should be explored.
\end{abstract}

Keywords: HIV positive adolescents and young adults, viral sexually transmitted infections, sub-Saharan Africa

\begin{abstract}
Abbreviations
ALHIV: Adolescents Living with HIV/ AIDS; ART: Antiretroviral Therapy; CSF: Civil Society Fund; HPV: Human Papilloma Virus; HSV: Herpes Simplex Virus; IDI: Infectious Diseases Institute; STIs: Sexually Transmitted Infections; YALHIV: Young Adults Living with HIV/AIDS
\end{abstract}

\section{Introduction}

Adolescents living with HIV (ALHIV) and young adults living with HIV (YALHIV) in Africa are at an increased risk of acquiring STIs. STIs can be due to parasitic, bacterial, fungal or viral agents. Widespread use of antimicrobials worldwide has led to a decline in the prevalence of bacterial STIs while the prevalence of viral STIs on the contrary, have been on the rise [1-3]. Viral STIs which include HPV-associated Condylomata acuminata, poxvirus-associated Molluscum contagiosum and HSV type I or II infections generally contribute to two-thirds of the total
STI burden hence are an important factor in HIV transmission [2,4].

Genital examinations are often overlooked during clinic consultations in many HIV care programs due to large patient loads and a lack of experienced staff. As a consequence, many patients with STIs are treated empirically with presumptive antimicrobial therapy at the initial evaluation, citing high STI positivity rates in HIV positive persons and low followup visit rates $[5,6]$.

Viral STIs particularly HSV disease have been shown to increase HIV transmission [79]. Up to $97 \%$ of HIV-infected individuals are co-infected with HSV-1, HSV-2, or both [10-12]. The clinical burden of viral STIs among ALHIV and YALHIV has not been separately described. We conducted a oneyear observational study in a large urban HIV clinic in sub-Saharan Africa to determine the clinical burden of specific viral STIs among ALHIV and YALHIV.

\section{Christine Katusiime*}

Department of Prevention, Care and Treatment, Infectious Diseases Institute College of Health Sciences, Makerere University, Kampala, Uganda

*Author for correspondence: katutina1@gmail.com 


\section{Methods}

\section{Study setting}

The Infectious Diseases Institute (IDI) is a center of excellence for HIV care and treatment located at the College of Health Sciences, Makerere University, Mulago National Referral Hospital in Kampala, Uganda. The out-patient clinic provides AntiRetroviral Therapy (ART) free of charge. The IDI established an adolescent/young adult HIV clinic in 2008 to provide adolescent/ young adult HIV/AIDS care to ALHIV and YALHIV aged 15-24 years, a successful attempt to bridge the gap between pediatric and adult HIV/AIDS care.

In April 2011, STI screening and management was incorporated into the adolescent and young adult HIV care package. Since its inception, the transition STI program has provided care to over 600 ALHIV and YALHIV. We sought to establish the prevalence of clinically diagnosed viral STIs over one study period.

From April 2017 to April 2018, symptomatic ALHIV and YALHIV were treated in the transition STI program by a dedicated adolescent STI clinician. For the purposes of this study, a diagnosis of a viral STI was established by clinical examination alone. We defined a positive viral STI outcome as a clinical diagnosis of Condylomata acuminata, Molluscum contagiosum or genital herpes made by the clinician. Condylomata acuminata (HPV) was differentiated from Condylomata lata (secondary syphilis) as having lesions that were pearly, fungating, cauliflower, plaque-like as compared to the latter that characteristically presents as elevated plaques, nodular lesions or maculopapular rash $[13,14]$.

Patients were identified by the triage nurses and referred to the adolescent STI clinician (FIGURE 1). Physical examinations were conducted by the adolescent STI clinician in the presence of an STI nurse/counselor. Data was entered into Epilnfo and analyzed using SPSS version 22.0.

\section{Results}

During the study period, a total of 165 ALHIV and YALHIV accessed treatment from the transition STI program. Eightyone $(49.1 \%)$ participants were clinically diagnosed with a viral STI, $72(88.9 \%)$ of whom were female (TABLE 1). Only one of the female participants reported being a commercial sex worker. None of the male participants who were diagnosed with a viral STI was circumcised. The median age of the patients was 22.0 years old (IQR: 20.0-23.0) and the median CD4+ count was 363 cells/ ul $(192-495$ cells $/ \mu \mathrm{l})$. Of the 81 patients

\section{Identification of symptomatic patients by triage nurses}
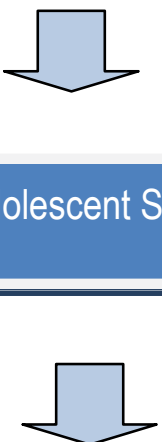

Genital examination 


\begin{tabular}{|c|c|c|}
\hline Characteristic & Female $\mathbf{n}(\%)$ & Male n (\%) \\
\hline Number & $72(88.9 \%)$ & $9(11.1 \%)$ \\
\hline Genital herpes & $50(87.7 \%)$ & $7(12.3 \%)$ \\
\hline Molluscum Contagiosum & $4(100 \%)$ & $0(0 \%)$ \\
\hline Condylomata Acuminata & $27(93.1 \%)$ & $2(6.9 \%)$ \\
\hline \multicolumn{3}{|l|}{ CD4 counts } \\
\hline$<350$ cells/ $\mu \mathrm{l}$ & $36(90 \%)$ & $4(10 \%)$ \\
\hline$>350$ cells $/ \mu \mathrm{l}$ & $36(87.8 \%)$ & $5(12.2 \%)$ \\
\hline \multicolumn{3}{|l|}{ WHO stage } \\
\hline I / II & $51(87.9 \%)$ & $7(12.1 \%)$ \\
\hline III / IV & $21(91.3 \%)$ & $2(8.7 \%)$ \\
\hline
\end{tabular}

diagnosed with a viral STI, only 19 (23.5\%) were on anti-retroviral therapy (ART) at the time of presentation to the clinic. Following the adoption of the test and treat policy, in November 2015, all ALHIV and YALHIV were initiated on ART and provided with STI treatment as well.

Condylomata acuminata were identified in $29 / 81(35.8 \%)$ participants (27 females/2 males, mean age: 21 years, range 16-24) and Molluscum contagiosum was identified in 4/81 (4.9\%) participants (all 4 participants were women, mean age: 24 years). Only one participant (female participant) was identified as having both Condylomata acuminata and Molluscum contagiosum infection.

HSV infection was diagnosed in $57 / 81$ (70.4\%) participants (50 females/7 males, mean age: 21 years, range 16-24). One male participant was diagnosed with recurrent genital herpes and was put on long-term suppressive antiviral therapy for 6 months in addition to ART. Simultaneous diagnosis of genital HSV infection and condylomata acuminata was observed in 6 participants; all of whom were female. Two female participants were diagnosed with both genital HSV infection and Molluscum contagiosum. None of the participants had clinical evidence of all three viral STIs at the time of examination.

The female participants diagnosed with viral STIs were more than the male participants by eight fold: $88.9 \%$ vs. $11.1 \%$. There was very strong evidence that ALHIV and YALHIV were more likely to have genital HSV disease than condylomata acuminata $\left(X^{2}=59.0 ; 1 \mathrm{df} ; \mathrm{p}<0.0001\right)$.

Acyclovir was prescribed for primary and recurrent HSV genital disease. Treatments for Condylomata acuminata and Molluscum contagiosum were not available in our clinic and patients were referred to the STD clinic at the Mulago National Referral Hospital for management of these conditions.

\section{Discussion}

To our knowledge, this is the first study demonstrating the epidemiological profile of clinically diagnosed viral STIs in an urban population of HIV sero-positive adolescents and young adults receiving HIV care in subSaharan Africa. The high burden of viral STIs among ALHIV and YALHIV highlights the need for HIV programs to incorporate rigorous STI management in adolescent/ young adult HIV care packages.

Female participants were more likely than their male counterparts to access STI services. Genital HSV disease was the most common viral STI $(70.4 \%)$ followed by Condylomata acuminata $(35.8 \%)$ in our adolescent cohort. These findings are in keeping with the recent findings from the Indian HIV cohort, were genital herpes was the most common viral infection followed by genital molluscum [15].

Our study has a number of limitations. All the STIs were not studied; the focus was only on clinically diagnosed viral STIs. HSV culture, PCR or serology, and HPV PCR were not available in our clinic because of the high costs involved. Acetoacid screening for HPV cervical pathology is also not routinely done. As a consequence, we are likely to have underestimated the burden of viral disease. The other limitation of our study is that the small number of study patients may affect its external validity.

The study demonstrates that almost half of ALHIV and YALHIV in our clinic were 
diagnosed with viral STIs hence underscoring the importance of routine genital examinations as part of STI screening in adolescent HIV care packages in resource-limited settings where HSV and HPV diagnostic tests may not be available.

Although this article illustrates that STIs are frequently identified among female ALHIV and YALHIV as compared to their male counterparts, it is critical that HIV programs offer dedicated STI care, specialized counseling and outreach services that encompass effective partner notification, tracking and treatment to male YALHIV and ALHIV.

\section{Conclusion}

Our study confirmed that genital herpes is the most common clinical viral STI in symptomatic ALHIV and YALHIV. Providers can expect that a significant proportion of ALHIV and YALHIV will require therapy for both acute episodes of genital herpes disease and recurrent disease. Genital examinations should, therefore, be part of routine adolescent and young adult HIV care packages. Future research interventions directed at developing single, dual or multiple point-of-care STI testing will go a long way to diagnosing asymptomatic viral STIs in this population.

\section{Funding}

This study was funded by the Civil Society Fund (CSF); grant number CSF/003/2017.
The author disclosed receipt of the following financial support for the research of this article: the Civil Society Fund (CSF) Uganda: grant number CSF/003/2017.

\section{Acknowledgment}

I accord double honor to the Chief Custodian of this project, Prophet Elvis Mbonye to whom I am completely and entirely indebted.

The author wishes to thank the patients and the sexual reproductive health staff for their contribution to this work.

\section{Competing Interest}

The authors declare that there are no competing interests.

\section{Ethics Approval and Consent To Participate}

The study protocol was approved by the institutional ethics review board at the Infectious Diseases Institute. Written consent from the patients was obtained prior to data collection.

\section{Author Contribution}

$\mathrm{CK}$ is the corresponding author; clinician; involved in the study, design, and analysis of data; drafted and critically revised the manuscript.

\section{Consent for Publication}

Not applicable. 


\section{References}

Sarkar S, Shrimal A, Das J, Choudhury S. Pattern of sexually transmitted infections: a profile from a sexually transmitted infections clinic of a tertiary care hospital of eastern India. Ann. Med. Health Sci. Res. 3(2), 206-209 (2013).

Vora R, Anjaneyan G, Doctor C, Gupta R. Clinico-epidemiological study of sexually transmitted infections in males at a rural-based tertiary care center. Indian J. Sex. Transm. Dis. 32(2), 86-89 (2011).

Sharma S, Tiwari S, Paliwal V, Mathur DK, Bhargava P. Study of patterns of sexually transmitted diseases using a syndromic approach in the era of human immunodeficiency virus from a tertiary care hospital of Northern India. Indian J. Sex Transm. Dis. 36(2), 158-161 (2015).

Arora P, Nagelkerke NJ, Jha P. A systematic review and meta-analysis of risk factors for sexual transmission of HIV in India. PLoS One. 7(8), e44094 (2012).

Sena AC, Hsu KK, Kellogg N, et al. Sexual assault and sexually transmitted infections in adults, adolescents, and children. Clin. Infect. Dis. 61, S856-S864
(2015). html

https://www.cdc.gov/std/tg2015/pid.

Horbul JE, Schmechel SC, Miller BR. Herpes simplex virus-induced epithelial damage and susceptibility to human immunodeficiency virus type 1 infection in human cervical organ culture. PLoS One. 6, e22638 (2011).

Barnabas V, Wasserheit JN, Huang Y, et al. Impact of herpes simplex virus type 2 on HIV-1 acquisition and progression in an HIV vaccine trial (the Step Study). J. Acquir. Immun. Defic. Syndr. 57, 238-244 (2011).

Barnabas RV, Webb EL, Weiss HA, Wasserheit JN. The role of coinfections in HIV epidemic trajectory and positive prevention: A systematic review and metaanalysis. AIDS. 25, 1559-1573 (2011).

Des Jarlais DC, Arasteh K, McKnight C, et al. HSV-2 co-infection as a driver of HIV transmission among heterosexual non-injecting drug users in New York City. PLoS One. 9(1), e87993 (2014).

Looker KJ, Elmes JAR, Gottlieb SL, et al. Effect of HSV-2 infection on subsequent HIV acquisition: an updated systematic review and meta-analysis. Lancet Infect. Dis. 17(12), 1303-1316 (2017).

Yap SH, Abdullah NK, McStea M, et al. HIV/Human herpesvirus co-infections: Impact on the tryptophan-kynurenine pathway and immune reconstitution. PLoS One. 12(10), e0186000 (2017).

Danescu SA, Szolga B, Georgiu C. Unusual manifestations of secondary syphilis: Case presentations. Acta Dermatovernerol Croat. 26(2), 186-188 (2018).

Leonard B, Kridelka F, Delbecque K, et al. A clinical and pathological overview of vulvar condyloma acuminatum, intraepithelial neoplasia and squamous cell carcinoma. Biomed. Res. Int. 480573 (2014).

Chugh S, Garg VK, Sarkar R, Sardana K. Clinico-epidemiological profile of viral sexually transmitted infections in seropositive patients attending a tertiary care hospital in North India. J. Int. Assoc. Provid. AIDS Care. 16(4), 331-337 (2017). 